\title{
A Cubic Autocatalator Chemical Reaction Model with Limit Cycle Analysis and Consistency Preserving Discretization
}

\author{
Qamar Dina,*, Muhammad Sajjad Shabir ${ }^{a}$, Muhammad Asif \\ Khan $^{\text {a }}$ \\ ${ }^{a}$ Department of Mathematics, University of Poonch Rawalakot, Azad Kashmir, Pakistan \\ qamar.sms@gmail.com, sajjadmust@gmail.com, asif31182@gmail.com
} (Received September 12, 2021)

\begin{abstract}
This article deals with the study of some qualitative properties of a cubic autocatalator chemical reaction model. Particularly, we obtain a dynamically consistent cubic autocatalator discrete-time model by applying a nonstandard difference scheme. Analysis of the existence of equilibria and their stability is carried out. It is proved that a continuous system undergoes the Hopf bifurcation at its interior equilibrium, whereas the discrete-time version undergoes NeimarkSacker bifurcation at its interior fixed point. Moreover, numerical simulation is provided to strengthen our theoretical discussion.
\end{abstract}

\section{Introduction}

Due to strong nonlinearity, there exists complexity in various chemical processes involving chemical reactions such as mass transfer and heat, fluid flow and separations. Process engineers obtained a product by specifying homogeneous properties to keep the conditions of process stable and under control. Due to the natural nonlinearity of the reaction, sudden complexity is encountered in the industrial process without any external disturbances. In the last decay, a

\footnotetext{
* Corresponding author.
} 
significant number of articles have been published for processes showing oscillatory behavior, multiple fixed points and chaos [37-39].

The study of oscillatory behavior of chemical reaction is of great interest of both experimentalists and theoreticians for over a hundred years. The most considerable example of oscillatory systems in chemical engineering and chemical thermodynamics include the Belousov-Zhabotinsky, Bray-Liebhafsky and Briggs-Rausher reactions, for which oscillatory behavior in concentration can be observed through changes in color [2]. In the literature, a commonly used reactor for the study of such chemical dynamics, is the continuous flow well-stirred tank reactor (CSTR). It is also known as backmix reactor or vat $[3,4]$. In experiments, CSTR governs the system of ordinary differential equations, which can be handled via standard techniques.

Moreover, numerous investigations illustrate that CSTRs may reveal a rich behavior in dynamic phenomena [36], through fluctuation being the feature that has been subjected to passionate research activity by both chemical engineers and mathematicians. The investigation of self-oscillatory CSTRs has evolved into two separate dictions: one is the removal of the oscillations and the other is to take advantage of the process dynamics for unsteady-state operation [37-39]. The optimal proposed model would extend beyond the conventional fixed-point optimization by involving the factor of time-evolution, which recommends chances for latent route enrichment by the cyclic process.

Chaos could be produced through a combination of two oscillatory CSTRs [40-42]. Complex dynamical behavior of CSTRs is explored by investigating bifurcation analysis of steady-state. Bifurcation analysis not only investigates the unforced system but also estimates the dynamic complexities when periodic forcing is introduced. Classic methodical tools include codimension-1 bifurcations of limit cycles and stroboscopic Poincare maps [43], due to chaos during period-doubling and Neimark-Sacker bifurcations being frequently evident in the CSTRs.

Mathematical modeling of the most well-known chemical reaction Bclousov-Zhabotinskil (BZ) reaction was the first time proposed by Field and Noyes [5] under isothermal conditions. Leach et al. [31], studied the two-cell coupled cubic autocatalator chemical reaction model and showed the existence of Hopf bifurcation. In [32], the author explored the existence of the limit cycle for a cubic chemical autocatalator model and evident that the limit cycles exist for a restricted region of parameter space. In [33], the author investigated a cubic autocatalator chemical reaction model and showed that traveling waves do not occur whenever the initial concentration of reactant is not periodic. In [34], the author 
studied a homogeneous-heterogeneous reaction of nano-fluid and showed the existence of hysteresis bifurcations and multiple solutions. Alderremy et al. [35], proposed a new fractional blood ethanol model and discussed a brief comparison with the fractional-cubic autocatalator reaction model. Kay and Scott [6] showed that these reactions also exhibit oscillation in exothermic reactions (see also [7, 8]). Gray and Scott [9] proposed a cubic autocatalator chemical reaction model that exhibits oscillatory behavior, and the proposed hypothetical reaction is governed by the following system:

$$
P \stackrel{k_{0}}{\rightarrow} A, 2 B \stackrel{k_{1}}{\rightarrow} 3 B, \quad B \stackrel{k_{2}}{\rightarrow} C, \quad A \stackrel{k_{3}}{\rightarrow} B
$$

where $k_{0}, k_{1}, k_{2}, k_{3}$ are constant quantities and $P, A, B$ and $C$ are some chemical species along with $p, a, b$ and $c$ are their molar concentrations respectively. The following system is obtained by assuming isothermal reaction;

$$
\begin{aligned}
& \frac{d a}{d t}=k_{0} p-k_{1} a b^{2}-k_{a} \\
& \frac{d b}{d t}=k_{1} a b^{2}-k_{2} b+k_{3} a .
\end{aligned}
$$

The dimensionless form of the system (2) can be expressed as (cf. [10]):

$$
\begin{aligned}
& \frac{d x}{d t}=\mu-x y^{2}-r x, \\
& \frac{d y}{d t}=x y^{2}-y+r x,
\end{aligned}
$$

where $r, \mu>0$. The detailed analysis of system (3) is carried out by Merkin et al. [11]. In [12], the authors discussed the special case of the model (3) with $r=0$ and showed the existence of periodic behavior for parameter $\mu$. Gray and Thuraisingham [13] modified the system (3) with addition of some extra parameters and discussed the bifurcation analysis. Moreover, Forbes and Holmes [10] discussed the limit cycle behavior of the system (3).

Notice that, due to rich dynamics and remarkable computing results in the discrete dynamical system is more suitable than continuous one $[14,15]$. Furthermore, in the case of non-linear chemical oscillatory reaction models, this argument works efficiently [16-18]. Therefore, we explored the stability analysis of the discrete-time version of the system (3) and bifurcation analysis. The investigation of such discrete-time chemical oscillatory model can be found in [1,19-23, 30,31].

According to Strogatz [24], chaos can exists in a 3-dimensional phase space continuous system at least. Therefore it is clear that in the system (3) chaos can not be observed. While in the case of the counter discrete-time map, chaos can be observed in one dimension.

Motivated by the aforementioned rich properties of discrete-time dynamical systems, it 
is necessary and interesting to study the qualitative behavior of the discrete-time version of the system (3). Therefore, by applying a non-standard finite difference scheme to the system (3), we have the following discrete-time cubic autocatalator chemical reaction model:

$$
\begin{aligned}
& x_{n+1}=\frac{\mu h+x_{n}}{1+h\left(r+y_{n}^{2}\right)}, \\
& y_{n+1}=\frac{y_{n}+h\left(r+y_{n}^{2}\right) x_{n}}{1+h},
\end{aligned}
$$

where $r, \mu, h$ are positive constants. The novel contributions of this manuscript are given below:

- Limit cycle analysis of system (3) is carried out by computing the first Lyapunov exponent (FLE).

- A novel discrete-time cubic autocatalator chemical reaction model (4) is obtained which is dynamically consistent.

- Parametric conditions are obtained for the qualitative behavior of the system (4) including stability analysis and Neimark-Sacker bifurcation about coexistence.

The remaining part of this manuscript organized as Andronov-Hopf bifurcation for system (3) is discussed in Section 2. The existence of equilibrium point and local stability of the model (4) is investigated in Section 3. Bifurcation analysis of the unique positive equilibrium point of the system (4) is discussed in Section 4. Finally, comprehensive numerical simulations are provided to support the main theoretical investigation in Section 5.

\section{Andronov-Hopf bifurcation in system (3)}

By performing simple algebraic manipulation, it is easy to see that systems (3) and (4) have unique positive equilibrium point $E=\left(\frac{\mu}{r+\mu^{2}}, \mu\right)$.

First, we see the dynamical behavior of the continuous system (3) at its unique positive equilibrium point. For this, the variational matrix of system (3) evaluated at $E=\left(\frac{\mu}{r+\mu^{2}}, \mu\right)$ is given by:

$$
V(E)=\left[\begin{array}{cc}
-r-\mu^{2} & -\frac{2 \mu^{2}}{r+\mu^{2}} \\
r+\mu^{2} & 1-\frac{2 r}{r+\mu^{2}}
\end{array}\right] .
$$

It is easy to see that $\operatorname{det} V(E)=r+\mu^{2}>0$ and $\operatorname{Tr} V(E)=1-\mu^{2}-r\left(1+\frac{2}{r+\mu^{2}}\right)$. Therefore, according to Routh-Hurwitz stability critearion, $\left(\frac{\mu}{r+\mu^{2}}, \mu\right)$ is a sink if and only if 
$1-\mu^{2}-r\left(1+\frac{2}{r+\mu^{2}}\right)<0$ and it is a source if and only if $1-\mu^{2}-r\left(1+\frac{2}{r+\mu^{2}}\right)>0$, and system (3) undergoes Hopf bifurcation if the parameters belong to the following curve:

$$
C_{H B}=\left\{(\mu, r) \in \mathbb{R}^{2}: r=\frac{1}{2}\left(-1-2 \mu^{2}+\sqrt{1+8 \mu^{2}}\right), r>0, \mu>0\right\} .
$$

Assume that $r, \mu \in C_{H B}$, then system (3) undergoes Hopf bifurcation. Furthermore, for $r \in$ $[0,0.3]$ and $\mu \in[0,1]$ the topological classification of the system (3) at its positive steady-state is depicted in Fig. 1. In Fig. 1, white region represents source and black region denotes sink.

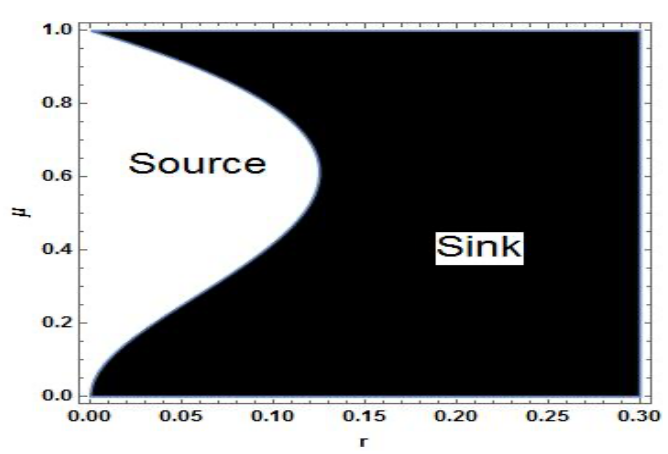

Figure 1. Topological classification for system (3).

In order to study the periodic nature of solutions of system (3), we discuss the appearance of supercritical and subcritical Hopf bifurcations in the system (3). For this, we consider a general autonomous planar system as follows:

$$
\left\{\begin{array}{l}
x^{\prime}(t)=f(\alpha, x, y) \\
y^{\prime}(t)=g(\alpha, x, y)
\end{array}\right.
$$

where $\alpha \in \mathbb{R}$ is bifurcation parameter. Assume that $\left(x^{*}, y^{*}\right)$ be any equilibrium point of (5), and $J\left(x^{*}, y^{*}\right)$ is the variational matrix of (5) evaluated at $\left(x^{*}, y^{*}\right)$. Furthermore, assume that eigenvalues of $J\left(x^{*}, y^{*}\right)$ are of the following form:

$$
\lambda_{1,2}(\alpha)=\psi(\alpha) \pm \iota \omega(\alpha) .
$$

Moreover, suppose that there exists a particular value of $\alpha$, say $\alpha_{0}$, such that the following conditions are satisfied:

(i) $\psi\left(\alpha_{0}\right)=0$, and $\omega\left(\alpha_{0}\right)=\omega_{0} \neq 0$, which is known as a non-hyperbolicity condition, that is, the existence of conjugate pair of imaginary eigenvalues for $J\left(x^{*}, y^{*}\right)$. 
(ii) $\left.\frac{d \psi(\alpha)}{d \alpha}\right|_{\alpha=\alpha_{0}}=T \neq 0$, which is known as transversality condition, that is, the eigenvalues of $J\left(x^{*}, y^{*}\right)$ cross the imaginary axis with non-zero speed.

(iii) There exists a discriminatory quantity $L\left(\alpha_{0}\right) \neq 0$, which is known as the first Lyapunov exponent (FLE) and is defined as follows:

$$
\begin{aligned}
L\left(\alpha_{0}\right) & :=\frac{1}{16}\left(f_{x x x}+f_{x y y}+g_{x x y}+g_{y y y}\right) \\
& +\frac{1}{16 \omega_{0}}\left(f_{x y}\left(f_{x x}+f_{y y}\right)-g_{x y}\left(g_{x x}+g_{y y}\right)-f_{x x} g_{x x}+f_{y y} g_{y y}\right),
\end{aligned}
$$

where $f_{x y}=\frac{\partial^{2} f(\alpha, x, y)}{\partial x \partial y}$ evaluated at $(x, y)=\left(x^{*}, y^{*}\right)$ and $\alpha=\alpha_{0}$, etc. Furthermore, condition (iii) is known as the generosity condition.

If conditions (i)-(iii) are satisfied, then there exists a unique curve of periodic solutions bifurcates from the equilibrium point into the region $\alpha>\alpha_{0}$ if $T L\left(\alpha_{0}\right)<0$ or $\alpha<\alpha_{0}$ if $T L\left(\alpha_{0}\right)>0$. The steady-state is stable for $\alpha>\alpha_{0}$ (resp. $\alpha<\alpha_{0}$ ) and unstable for $\alpha<$ $\alpha_{0}$ (resp. $\alpha>\alpha_{0}$ ) if $T<0$ (resp. $T>0$ ) whereas the periodic solutions are stable (resp. unstable) if the fixed point is unstable (resp. stable) on the side of $\alpha=\alpha_{0}$ where the periodic solutions exist. Then, the amplitude of the periodic orbits grows like $\sqrt{\left|\alpha-\alpha_{0}\right|}$ and their periods tend to $\frac{2 \pi}{\left|\omega_{0}\right|}$ as $\alpha$ approaches to $\alpha_{0}$. The bifurcation is called supercritical if the bifurcating periodic solutions are stable, and subcritical if they are unstable.

Keeping in view the above discussion for Andronov-Hopf bifurcation, we consider the system (3). For this, we suppose that $4\left(r+\mu^{2}\right)^{3}-\left(r(1+r)+(2 r-1) \mu^{2}+\mu^{4}\right)^{2}>0$, then it is easy to see that the eigenvalues of variational matrix $V(E)$ are of the form:

$$
\lambda_{1,2}(r)=\psi(r) \pm \iota \omega(r)
$$

where

$$
\psi(r)=-\frac{r+r^{2}-\mu^{2}+2 r \mu^{2}+\mu^{4}}{2\left(r+\mu^{2}\right)}
$$

And

$$
\omega(r)=\frac{\sqrt{4\left(r+\mu^{2}\right)^{3}-\left(r(1+r)+(2 r-1) \mu^{2}+\mu^{4}\right)^{2}}}{2\left(r+\mu^{2}\right)} .
$$

Next, $\psi(r)=0$ gives $r=\frac{1}{2}\left(-1-2 \mu^{2} \pm \sqrt{1+8 \mu^{2}}\right)$. Neglecting the negative value of $r$ we left with $r \equiv r_{0}=\frac{1}{2}\left(-1-2 \mu^{2}+\sqrt{1+8 \mu^{2}}\right)$ such that $0<\mu<1$. At $r=r_{0}$, one has $\omega\left(r_{0}\right) \equiv \omega_{0}=\sqrt{r_{0}+\mu^{2}} \neq 0$. For transversality condition, we see that 


$$
\left.\frac{d \psi(r)}{d r}\right|_{r=r_{0}}=\frac{1}{\frac{1}{\sqrt{1+8 \mu^{2}}}-1}<0 .
$$

In order to shift the equilibrium of system $(3)$ at $(0,0)$, we consider the following translations:

$$
u(t)=x(t)-x^{*}, v(t)=y(t)-y^{*},
$$

where $x^{*}=\frac{\mu}{r+\mu^{2}}$ and $y^{*}=\mu$. With implementing this translation to the system (3) yields the following planar system:

$$
\begin{aligned}
& \frac{d u}{d t}=\mu-\left(u+x^{*}\right)\left(v+y^{*}\right)^{2}-r\left(u+x^{*}\right), \\
& \frac{d v}{d t}=\left(u+x^{*}\right)\left(v+y^{*}\right)^{2}-\left(v+y^{*}\right)+r\left(u+x^{*}\right) .
\end{aligned}
$$

An application of Taylor series expansion about $(u, v)=(0,0)$ gives the following system:

$$
\left(\begin{array}{l}
\frac{d u}{d t} \\
\frac{d v}{d t}
\end{array}\right)=A\left(\begin{array}{l}
u \\
v
\end{array}\right)+\left(\begin{array}{c}
-u v^{2}-2 u v \mu-\frac{v^{2} \mu}{r_{0}+\mu^{2}} \\
u v^{2}+2 u v \mu+\frac{v^{2} \mu}{r_{0}+\mu^{2}}
\end{array}\right)
$$

where

$$
A=\left[\begin{array}{cc}
-r_{0}-\mu^{2} & -\frac{2 \mu^{2}}{r_{0}+\mu^{2}} \\
r_{0}+\mu^{2} & r_{0}+\mu^{2}
\end{array}\right]
$$

Next, we want to convert the matrix $A$ into its canonical form. For this the following similarity transformation is considered:

$$
\left(\begin{array}{l}
u(t) \\
v(t)
\end{array}\right)=\left[\begin{array}{cc}
1 & 0 \\
-\frac{\left(r_{0}+\mu^{2}\right)^{2}}{2 \mu^{2}} & -\frac{\left(r_{0}+\mu^{2}\right)^{3 / 2}}{2 \mu^{2}}
\end{array}\right]\left(\begin{array}{c}
w(t) \\
z(t)
\end{array}\right) .
$$

From (7) and (8), it follows that

$$
\left(\begin{array}{l}
\frac{d w}{d t} \\
\frac{d z}{d t}
\end{array}\right)=\left[\begin{array}{cc}
0 & \omega_{0} \\
-\omega_{0} & 0
\end{array}\right]\left(\begin{array}{l}
w \\
z
\end{array}\right)+\left(\begin{array}{l}
f(w, z) \\
g(w, z)
\end{array}\right),
$$

where

$$
\begin{gathered}
f(w, z)=-v\left(u(v+2 \mu)-\frac{v \mu}{r_{0}+\mu^{2}}\right) \\
g(w, z)=\frac{v\left(\left(r_{0}+\mu^{2}\right)^{2}-2 \mu^{2}\right)\left(r_{0} u(v+2 \mu)+\mu\left(v+u v \mu+2 u \mu^{2}\right)\right)}{\left(r_{0}+\mu^{2}\right)^{5 / 2}} \\
u=w, \quad v=-\left(\frac{\left(r_{0}+\mu^{2}\right)^{2}}{2 \mu^{2}}\right) w-\left(\frac{\left(r_{0}+\mu^{2}\right)^{3 / 2}}{2 \mu^{2}}\right) z
\end{gathered}
$$

Then, the first Lyapunov exponent for system (3) is computed as follows: 


$$
\begin{aligned}
& L\left(r_{0}\right):=\frac{1}{16}\left(f_{x x x}+f_{x y y}+g_{x x y}+g_{y y y}\right) \\
& \quad+\frac{1}{16 \omega_{0}}\left(f_{x y}\left(f_{x x}+f_{y y}\right)-g_{x y}\left(g_{x x}+g_{y y}\right)-f_{x x} g_{x x}+f_{y y} g_{y y}\right) .
\end{aligned}
$$

After some tedious calculations, the closed form of $L\left(r_{0}\right)$ is given by:

$$
L\left(r_{0}\right)=\frac{3\left(\sqrt{1+8 \mu^{2}}-1\right)-4 \mu^{2}\left(1+\sqrt{1+8 \mu^{2}}\right)}{32 \mu^{2}} .
$$

Moreover, we have $L\left(r_{0}\right)>0$ if and only if $0<\mu<\mu_{0}$ and $L\left(r_{0}\right)<0$ if and only if $\mu>$ $\mu_{0}$, where $\mu_{0}=\frac{\sqrt{3-\sqrt{6}}}{2} \cong 0.370981892151363$. Moreover, a plot of FLE in the unit interval is depicted in Fig. 2.

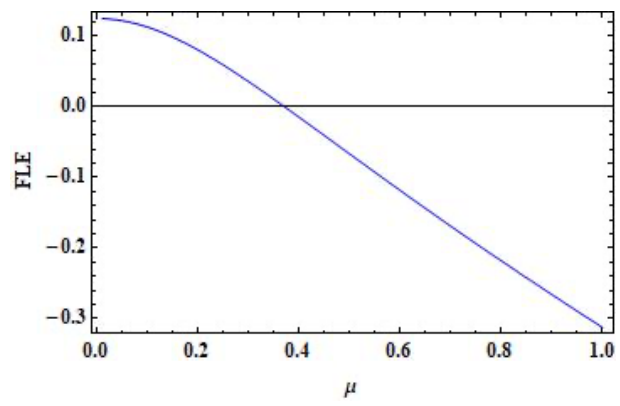

Figure 2. Plot of the first Lyapunov exponent.

\section{Stability analysis of system (4)}

In order to study the behavior of the model (4) at $\left(\frac{\mu}{r+\mu^{2}}, \mu\right)$, we assume that $F_{J}(E)$ be the variational matrix of model (4) at point $\left(\frac{\mu}{r+\mu^{2}}, \mu\right)$, then it follows that:

$$
F_{J}(E)=\left(\begin{array}{ll}
\frac{1}{1+h\left(r+\mu^{2}\right)} & -\frac{2 h \mu^{2}}{\left(r+\mu^{2}\right)\left(1+h\left(r+\mu^{2}\right)\right)} \\
\frac{h\left(r+\mu^{2}\right)}{1+h} & \frac{1+\frac{2 h \mu^{2}}{r+\mu^{2}}}{1+h}
\end{array}\right) .
$$

Then characteristic polynomial of the matrix $F_{J}(E)$ is given by:

$$
\mathbb{F}(\omega)=\omega^{2}-\left(\frac{1+\frac{2 h \mu^{2}}{r+\mu^{2}}}{1+h}+\frac{1}{1+h\left(r+\mu^{2}\right)}\right) \omega+\frac{\frac{2 h \mu^{2}}{r+\mu^{2}}+\frac{1}{1+h\left(r+\mu^{2}\right)}}{1+h}
$$

Furthermore, from (10) it is easy to see that:

$$
\mathbb{F}(1)=\frac{h^{2}\left(r+\mu^{2}\right)}{(1+h)\left(1+h\left(r+\mu^{2}\right)\right)^{\prime}}
$$


$\mathbb{F}(-1)=\frac{4 r+2 h r+2 h r^{2}+h^{2} r^{2}+4 \mu^{2}+6 h \mu^{2}+4 h r \mu^{2}+6 h^{2} r \mu^{2}+2 h \mu^{4}+5 h^{2} \mu^{4}}{\left(r+\mu^{2}\right)\left(1+h r+h \mu^{2}\right)}$,

and

$$
\mathbb{F}(0)=\frac{r+\mu^{2}+2 h \mu^{2}+2 h^{2} r \mu^{2}+2 h^{2} \mu^{4}}{(1+h)\left(r+\mu^{2}\right)\left(1+h r+h \mu^{2}\right)} .
$$

From (11) and (12), it follows that $\mathbb{F}(1)>0$ and $\mathbb{F}(-1)>0$, therefore according to Jury condition fixed point $\left(\frac{\mu}{r+\mu^{2}}, \mu\right)$ of the system (4) is a sink if and only if $\mathbb{F}(0)<1$. The dynamics of the system (4) with respect to its fixed point $\left(\frac{\mu}{r+\mu^{2}}, \mu\right)$ is summarized as follows. Theorem 3.1 For the fixed point $E=\left(\frac{\mu}{r+\mu^{2}}, \mu\right)$ of system (4), the following results are satisfied:

(i) $E=\left(\frac{\mu}{r+\mu^{2}}, \mu\right)$ is a sink if and only if $\mu^{2}\left(1+h \mu^{2}\right)<r\left(1+r+h r+2 \mu^{2}\right)+\mu^{4}$.

(ii) $E=\left(\frac{\mu}{r+\mu^{2}}, \mu\right)$ can not be a saddle point.

(iii) $E=\left(\frac{\mu}{r+\mu^{2}}, \mu\right)$ is unstable and source if and only if $\mu^{2}\left(1+h \mu^{2}\right)>r(1+r+h r+$ $\left.2 \mu^{2}\right)+\mu^{4}$.

(iv) $E=\left(\frac{\mu}{r+\mu^{2}}, \mu\right)$ is non-hyperbolic equilibrium point if and only if

$$
\frac{r+\mu^{2}+2 h \mu^{2}}{(1+h)\left(r+\mu^{2}\right)}+\frac{1}{1+h\left(r+\mu^{2}\right)}<2
$$

and

$$
\mu^{2}\left(1+h \mu^{2}\right)=r\left(1+r+h r+2 \mu^{2}\right)+\mu^{4} .
$$

Moreover, for $h=0.1$ the topological classification for fixed-point $E=\left(\frac{\mu}{r+\mu^{2}}, \mu\right)$ of the system (4) is depicted in Fig. 3 in $\mu r$-plane. In Fig. 3, white and black regions represent source and sink, respectively.

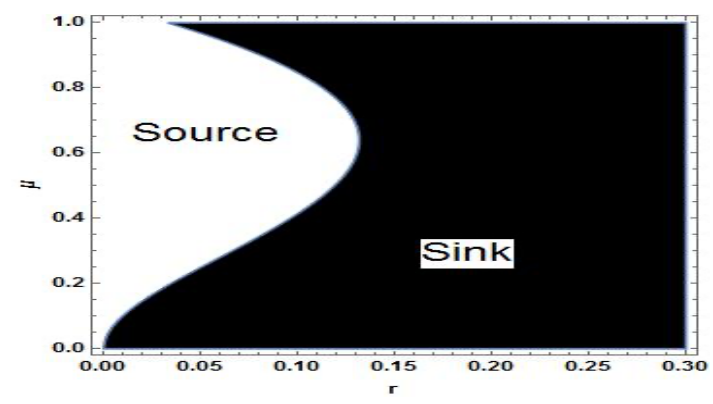

Figure 3. Topological classification of system (4). 


\section{Neimark-Sacker bifurcation in system (4)}

According to Theorem 3.1, the characteristic equation (10) has two complex conjugate roots with modulus one, if condition (iv) of Theorem 3.1 is satisfied. Hence, the unique positive equilibrium point of the model (4) undergoes Neimark-Sacker bifurcation if parameters vary in the neighborhood of the following set:

$$
B_{S}=\left\{(r, \mu, h): \mathrm{h} \equiv h_{1}=1+\frac{2 r}{\mu^{2}-r}-\frac{1}{r+\mu^{2}}, r, \mu, \mathrm{h}>0\right\} .
$$

Let $\left(r, \mu, h_{1}\right) \in B_{S}$ then the system (4) can be written as;

$$
\left(\begin{array}{l}
x \\
y
\end{array}\right) \rightarrow\left(\begin{array}{l}
\frac{\mu h_{1}+x}{1+h_{1}\left(r+y^{2}\right)} \\
\frac{y+h_{1}\left(r+y^{2}\right) x}{1+h_{1}}
\end{array}\right),
$$

where $h_{1}=1+\frac{2 r}{\mu^{2}-r}-\frac{1}{r+\mu^{2}}$. Let $|\tilde{h}| \ll 1$ be a perturbation parameter, then map (15) can be expressed as:

$$
\left(\begin{array}{l}
x \\
y
\end{array}\right) \rightarrow\left(\begin{array}{l}
\frac{\mu\left(h_{1}+\widetilde{h}\right)+x}{1+\left(h_{1}+\widetilde{h}\right)\left(r+y^{2}\right)} \\
\frac{y+\left(h_{1}+\widetilde{h}\right)\left(r+y^{2}\right) x}{1+\left(h_{1}+\widetilde{h}\right)}
\end{array}\right) .
$$

Next, under transformations $(H, P)=\left(x-\frac{\mu}{r+\mu^{2}}, y-\mu\right)$, the model (15) can be described by the following system:

$$
\left(\begin{array}{l}
H \\
P
\end{array}\right) \rightarrow\left(\begin{array}{ll}
Z_{11} & Z_{12} \\
Z_{21} & Z_{22}
\end{array}\right)\left(\begin{array}{l}
H \\
P
\end{array}\right)+\left(\begin{array}{l}
h_{1}(H, P) \\
h_{2}(H, P)
\end{array}\right)
$$

where

$$
\begin{aligned}
& h_{1}(H, P)=z_{13} H P+z_{14} H P^{2}+z_{15} P^{2}+k_{1} P^{3}+O\left((|H|+|P|)^{4},\right. \\
& h_{2}(H, P)=z_{23} H P+z_{24} H P^{2}+O\left((|H|+|P|)^{4},\right. \\
& z_{11}=\frac{1}{1+\left(h_{1}+\widetilde{h}\right)\left(r+\mu^{2}\right)}, z_{12}=-\frac{2\left(h_{1}+\widetilde{h}\right) \mu^{2}}{\left(r+\mu^{2}\right)\left(1+\left(h_{1}+\widetilde{h}\right)\left(r+\mu^{2}\right)\right)^{\prime}} \\
& z_{21}=\frac{\left(h_{1}+\widetilde{h}\right)\left(r+\mu^{2}\right)}{1+\left(h_{1}+\widetilde{h}\right)}, z_{22}=\frac{1+\frac{2\left(h_{1}+\widetilde{h}\right) \mu^{2}}{r+\mu^{2}}}{1+\left(h_{1}+\widetilde{h}\right)}, \\
& z_{13}=\frac{-2\left(h_{1}+\widetilde{h}\right) \mu}{\left(1+\left(h_{1}+\widetilde{h}\right)\left(\mu^{2}+r\right)\right)^{2}}, z_{14}=\frac{\left(\left(h_{1}+\widetilde{h}\right)\left(3 \mu^{2}-r\right)-1\right)\left(h_{1}+\widetilde{h}\right)}{\left(\left(h_{1}+\widetilde{h}\right)\left(\mu^{2}+r\right)+1\right)^{3}},
\end{aligned}
$$




$$
\begin{aligned}
& z_{15}=\frac{\left(h_{1}+\widetilde{h}\right) \mu\left(\left(h_{1}+\widetilde{h}\right)\left(3 \mu^{2}-r\right)-1\right)}{\left(\mu^{2}+r\right)\left(1+\left(h_{1}+\widetilde{h}\right)\left(\mu^{2}+r\right)\right)^{2}}, k_{1}=-4 \frac{\left(h_{1}+\widetilde{h}^{2} \mu^{2}\left(\left(h_{1}+\widetilde{h}\right)\left(\mu^{2}-r\right)-1\right)\right.}{\left(\mu^{2}+r\right)\left(\left(h_{1}+\widetilde{h}\right)\left(\mu^{2}+r\right)+1\right)^{3}}, \\
& z_{23}=2 \frac{h \mu}{1+\left(h_{1}+\widetilde{h}\right)}, z_{24}=\frac{\left(h_{1}+\widetilde{h}\right)}{1+\left(h_{1}+\widetilde{h}\right)} .
\end{aligned}
$$

The characteristic equation of the Jacobian matrix of the map (17) computed at $(0,0)$ can be described as follows:

$$
\omega^{2}-M_{1}(\tilde{h}) \omega+M_{2}(\tilde{h})=0,
$$

where

$$
M_{1}(\tilde{h})=\frac{r+\left(1+2\left(h_{1}+\tilde{h}\right)\right) \mu^{2}}{\left(1+\left(h_{1}+\widetilde{h}\right)\right)^{2}\left(r+\mu^{2}\right)}+\frac{1}{1+\left(h_{1}+\widetilde{h}\right)\left(r+\mu^{2}\right)}
$$

and

$$
M_{2}(\tilde{h})=\frac{\frac{2\left(h_{1}+\widetilde{h}\right) \mu^{2}}{r+\mu^{2}}+\frac{1}{1+\left(h_{1}+\widetilde{h}\right)\left(r+\mu^{2}\right)}}{1+\left(h_{1}+\widetilde{h}\right)}
$$

Since $\left(r, \mu, h_{1}\right) \in B_{S}$ and equation (18) has pair of complex conjugate roots with unit modulus then we have;

$$
\omega_{1}, \omega_{2}=\frac{M_{1}(\widetilde{h})}{2} \pm \frac{i}{2} \sqrt{4 M_{2}(\tilde{h})-M_{1}^{2}(\tilde{h})}
$$

Therefore we have

$$
\left|\omega_{1}\right|=\left|\omega_{2}\right|=\sqrt{M_{2}(\tilde{h})},\left(\frac{d \sqrt{M_{2}(\widetilde{h})}}{d \widetilde{h}}\right)_{\widetilde{h}=0}=\frac{2-\frac{2 r}{r+\mu^{2}}-\frac{1}{\left(1+\left(r+\mu^{2}\right) h_{1}\right)^{2}}-\frac{\left(r+\mu^{2}\right)\left(1+2 h_{1}\right)}{\left(1+\left(r+\mu^{2}\right) h_{1}\right)^{2}}}{2\left(1+h_{1}\right)^{2} \sqrt{\frac{2 \mu^{2} h_{1}}{r+\mu^{2}+\frac{1}{1+\left(r+\mu^{2}\right) h_{1}}}}} \neq 0 .
$$

Since $\left(r, \mu, h_{1}\right) \in B_{S}$, this implies that $-2<M_{1}(0)<2$. Thus $M_{1}(0) \neq \pm 2,0,1$ gives $\omega_{1}^{m}, \omega_{2}^{m} \neq 1$ for all $m=1,2,3,4$ at $\tilde{h}=0$. Hence, zeros of (18) do not lie in the intersection of the unit circle with the coordinate axes at $\tilde{h}=0$ and if the following condition is true:

$$
\frac{2 h_{1} \mu^{2}}{r+\mu^{2}}+\frac{1}{1+h_{1}\left(r+\mu^{2}\right)} \neq 0, \frac{1}{1+h_{1}\left(r+\mu^{2}\right)} \neq-\left(1+h_{1}\right) \frac{2 h_{1} \mu^{2}}{r+\mu^{2}} .
$$

Assume that $\gamma=\frac{M_{1}(0)}{2}, \delta=\frac{1}{2} \sqrt{4 M_{2}(0)-M_{1}^{2}(0)}$, then the normal form of (17) at $\tilde{h}=0$ can be expressed as:

$$
\left(\begin{array}{l}
H \\
P
\end{array}\right)=\left(\begin{array}{ll}
Z_{12} & 0 \\
\gamma-z_{11} & -\delta
\end{array}\right)\left(\begin{array}{l}
u \\
v
\end{array}\right) .
$$

hence, by using map (20) we get:

$$
\left(\begin{array}{l}
u \\
v
\end{array}\right) \rightarrow\left(\begin{array}{ll}
\gamma & -\delta \\
\delta & \gamma
\end{array}\right)\left(\begin{array}{l}
u \\
v
\end{array}\right)+\left(\begin{array}{l}
\widetilde{g_{1}}(u, v) \\
\widetilde{g_{2}}(u, v)
\end{array}\right)
$$


where

$$
\begin{aligned}
& \widetilde{g_{1}}(u, v)=\frac{z_{14}}{z_{12}} H P^{2}+\frac{z_{13}}{z_{12}} H P+\frac{z_{15}}{z_{12}} P^{2}+\frac{k_{1}}{z_{12}} P^{2}+O\left((|u|+|v|)^{4}\right), \\
& \widetilde{g_{2}}(u, v)=\left(\frac{\left(\gamma-z_{11}\right) z_{14}}{z_{12} \delta}-\frac{z_{24}}{\delta}\right) H P^{2}+\left(\frac{\left(\gamma-z_{11}\right) z_{13}}{z_{12} \delta}-\frac{z_{23}}{\delta}\right) H P+\frac{\left(\gamma-z_{11}\right) k_{1} P^{3}}{z_{12} \delta} \\
& +\frac{\left(\gamma-z_{11}\right) z_{15} P^{2}}{z_{12} \delta}+O\left((|u|+|v|)^{4}\right),
\end{aligned}
$$

$H=z_{12} u$ and $P=\left(\gamma-z_{11}\right) u-\delta v$. Therefore, we define the following nonzero real number:

$$
\mathrm{F}=\left(\left[-\operatorname{Re}\left(\frac{\left(1-2 \omega_{1}\right) \omega_{2}^{2}}{1-\omega_{1}} \tau_{20} \tau_{11}\right)-\frac{1}{2}\left|\tau_{11}\right|^{2}-\left|\tau_{02}\right|^{2}+\operatorname{Re}\left(\omega_{2} \tau_{21}\right)\right]\right)_{\widetilde{h}=0},
$$

where

$$
\begin{aligned}
& \tau_{20}=\frac{1}{8}\left[{\widetilde{g_{1}}}_{u u}-{\widetilde{g_{1}}}_{v v}+2{\widetilde{g_{2}}}_{u v}+i\left({\widetilde{g_{2}}}_{u u}-{\widetilde{g_{2}}}_{v v}-2{\widetilde{g_{1}}}_{u v}\right)\right], \\
& \tau_{11}=\frac{1}{4}\left[\widetilde{g_{1 u u}}+\widetilde{g_{1} v v}+i\left(\widetilde{g_{2 u u}}+\widetilde{g_{2 v}}\right)\right] \\
& \tau_{02}=\frac{1}{8}\left[{\widetilde{g_{1}}}_{u u}-{\widetilde{g_{1}}}_{v v}-2{\widetilde{g_{2}}}_{u v}+i\left({\widetilde{g_{2}}}_{u u}-{\widetilde{g_{2}}}_{v v}+2{\widetilde{g_{1}}}_{u v}\right)\right] \\
& \tau_{21}=\frac{1}{16}\left[{\widetilde{g_{1}}}_{u u u}+{\widetilde{g_{1}}}_{u v v}+{\widetilde{g_{2}}}_{u u v}+{\widetilde{g_{2}}}_{v v v}+i\left({\widetilde{g_{2}}}_{u u u}+{\widetilde{g_{2}}}_{u v v}-{\widetilde{g_{1}}}_{u u v}-{\widetilde{g_{1}}}_{v v v}\right)\right] .
\end{aligned}
$$

With bifurcation theory presented in ([25] - [29]), and taking into account the aforementioned computations, the following result is given for direction and existence of Neimark-Sacker bifurcation.

Theorem 3.1 There exists Neimark-Sacker bifurcation at $\left(\frac{\mu}{r+\mu^{2}}, \mu\right)$ whenever $h$ varies in a small neighborhood of $h_{1}=1+\frac{2 r}{\mu^{2}-r}-\frac{1}{r+\mu^{2}}$. In addition, if $\mathrm{F}<0,(\mathrm{~F}>0)$, respectively, then an attracting or repelling invariant closed curve bifurcates from the equilibrium point for $h>$ $h_{1}\left(h<h_{1}\right)$, respectively.

\section{Numerical simulation and discussion}

The present part of the manuscript is related to verification of the above theoretical work and validation of dynamical consistency of systems (3) and (4).

Example 5.1 First, we the assume special case of the system (1) and (4) by taking parameters $(\mu, r)=\left(0.9003, \frac{1}{8}\right)$, and initial values $\left(x_{0}, y_{0}\right)=(0.96,0.90)$, where the step size $h \in(0,1)$ 
for system (4) is taking as a bifurcation parameter, then the system (4) undergoes Neimark-Sacker bifurcation. Fig. 4 and Fig. 5 shows that both chemical concentrations undergo Neimark-Sacker bifurcation and corresponding maximum Lyapunov exponents are shown in Fig. 6. Particularly, when $h=0.295775$ then unique positive fixed point $E=$ $(0.962332,0.9003)$, undergoes Neimrk-Sacker bifurcation. Finally, some phase portraits are given in Fig. 7 to Fig. 10 for $0<h<1$.

Next, the characteristic polynomial of the system (4) computed at fixed point $E=$ $(0.962332,0.9003)$ is given by

$$
\mathbb{F}(\omega)=\omega^{2}-1.950527599147959 \omega+0.9999999999999999 .
$$

Then roots of $\mathbb{F}(\omega)=0$ are $\omega_{1,2}=0.9752637995739795 \pm 0.22104416129028326 i$ with $\left|\omega_{1,2}\right|=1$ so that $(\mu, r, h)=\left(0.9003, \frac{1}{8}, 0.29577469361743874\right) \in B_{S}$. Next, we observe that $M_{1}(0)=1.95053$ thus the condition (19) is satisfied. Moreover, some careful calculation gives

$$
\begin{aligned}
& \tau_{20}=\frac{1}{8}\left[{\widetilde{g_{1}}}_{u u}-{\widetilde{g_{1}}}_{v v}+2 \widetilde{g}_{2 u v}+i\left(\widetilde{g_{2}} u u-\widetilde{g_{2}} v-2 \widetilde{g_{1}} u v\right)\right]=-0.0231224+0.00970326 i, \\
& \tau_{11}=\frac{1}{4}\left[{\widetilde{g_{1}}}_{u u}+{\widetilde{g_{1}}}_{v v}+i\left({\widetilde{g_{2}}}_{u u}+\widetilde{g}_{2 v v}\right)\right]=-0.0254418+0.00696268 i, \\
& \tau_{02}=\frac{1}{8}\left[{\widetilde{g_{1}}}_{u u}-{\widetilde{g_{1}}}_{v v}-2{\widetilde{g_{2}}}_{u v}+i\left(\widetilde{g_{2}} \widetilde{g u}_{g_{v v}}+2 \widetilde{g_{1}} u v\right)\right]=-0.00907315+0.0399482 i \text {, } \\
& \tau_{21}=\frac{1}{16}\left[{\widetilde{g_{1}}}_{u u u}+{\widetilde{g_{1}}}_{u v v}+{\widetilde{g_{2}}}_{u u v}+{\widetilde{g_{2}}}_{v v v}+i\left({\widetilde{g_{2}}}_{u u u}+{\widetilde{g_{2}}}_{u v v}-{\widetilde{g_{1}}}_{u u v}-{\widetilde{g_{1}}}_{v v v}\right)\right] \\
& =-0.00488224+0.00339422 i \text {, }
\end{aligned}
$$

and

$$
\mathrm{F}=\left(\left[-\operatorname{Re}\left(\frac{\left(1-2 \omega_{1}\right) \omega_{2}^{2}}{1-\omega_{1}} \tau_{20} \tau_{11}\right)-\frac{1}{2}\left|\tau_{11}\right|^{2}-\left|\tau_{02}\right|^{2}+\operatorname{Re}\left(\omega_{2} \tau_{21}\right)\right]\right)_{\tilde{c}=0}=
$$

$-0.00383086<0$. This proves the correctness of Theorem 3.1 .

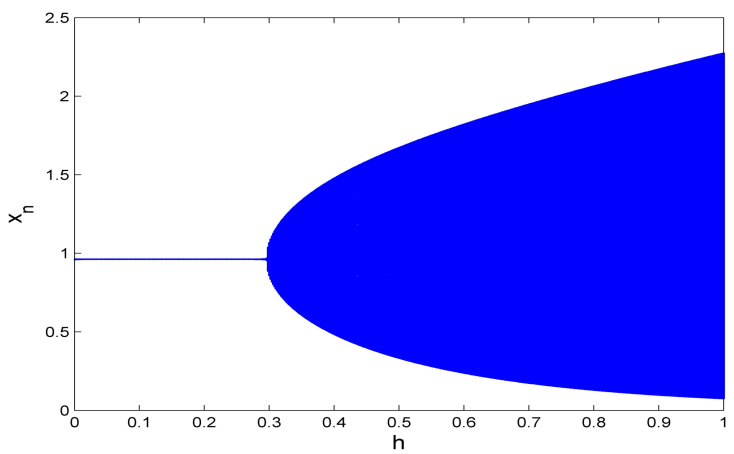

Figure 4. Bifurcation diagram for $x_{n}$ 


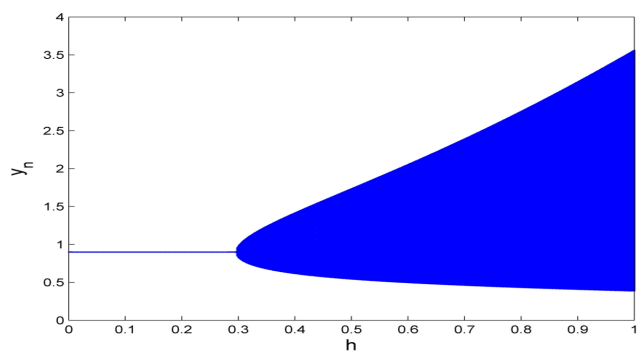

Figure 5. Bifurcation diagram for $y_{n}$

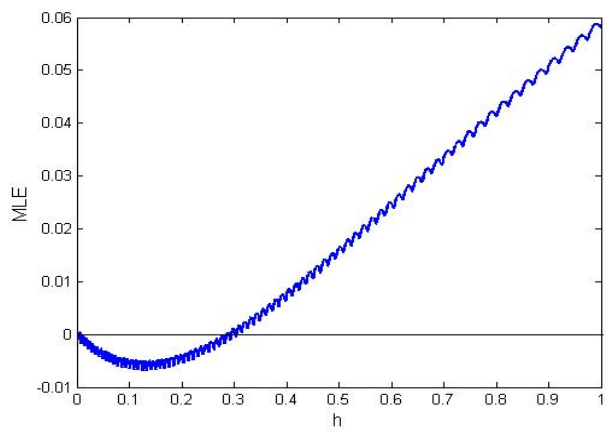

Figure 6. Maximum Lyapunov exponents

Bifurcation diagrams and MLE for the system (4) with $h \in(0,1)(r, \mu)=\left(\frac{1}{8}, 0.9003\right)$ and initial conditions $x_{0}=0.962332, y_{0}=0.9003$

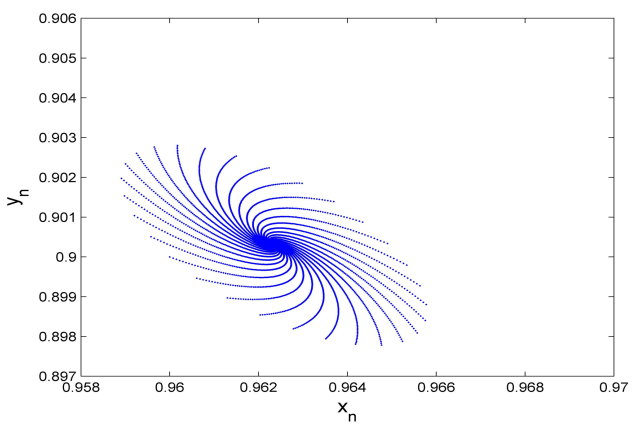

Figure 7. Phase portrait for system (4) 


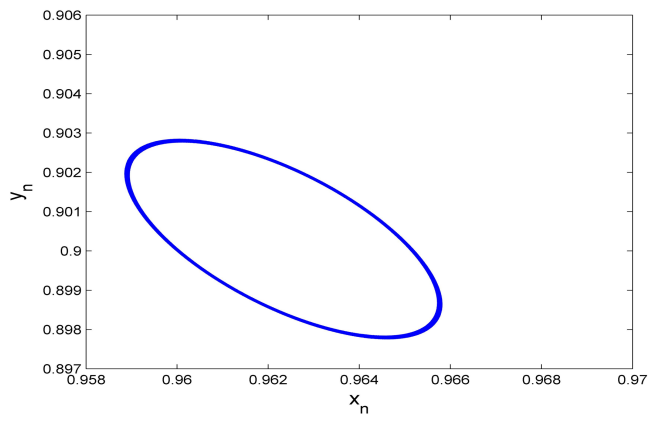

Figure 8. Phase portrait for system (4)

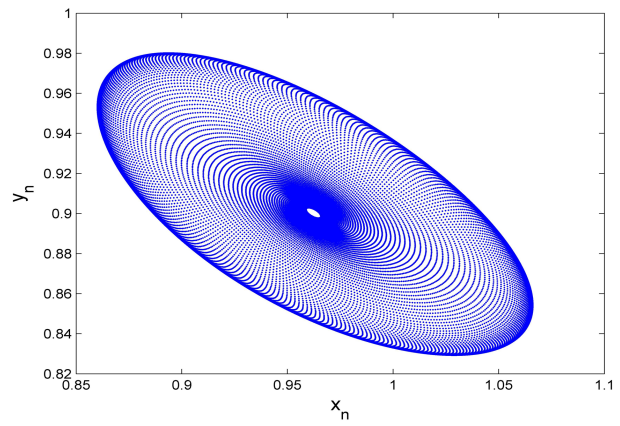

Figure 9. Phase portrait for system (4)

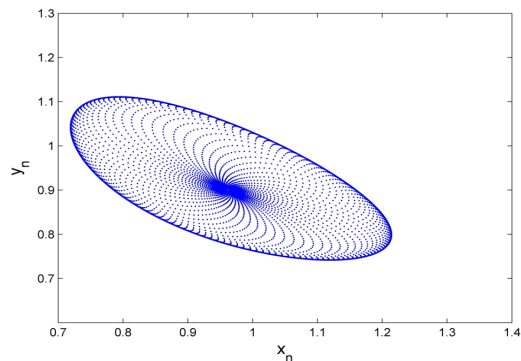

Figure 10. Phase portrait for system (4)

Phase portrait for the system (4) for $h \in(0,1)$ with $(r, \mu)=\left(\frac{1}{8}, 0.9003\right)$ and initial conditions $x_{0}=0.962332, y_{0}=0.9003$ 
Secondly, we take $(\mu, r)=\left(0.9003, \frac{1}{8}\right)$ in continuous system (3), then unique positive equilibrium $(0.962332,0.9003)$ is a sink. Indeed, the Jacobian matrix about these parametric values for system (3) is given as follows:

$$
\left[\begin{array}{cc}
-0.9355400899999999 & -1.7327746799177788 \\
0.9355400899999999 & 0.7327746799177788
\end{array}\right] \text {. }
$$

Consequently, the eignvalues are $-0.1013827050411106+0.961905212127758 i$ and $-0.1013827050411106-0.961905212127758 i$ both with negative real parts. The asymptotic stability of positive equilibrium is depicted in Fig. 11.

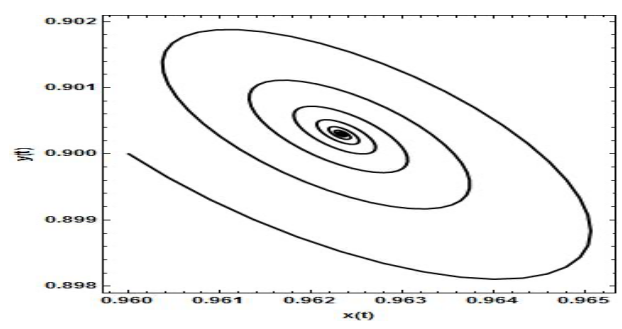

(a) Phase portrait for system (3)

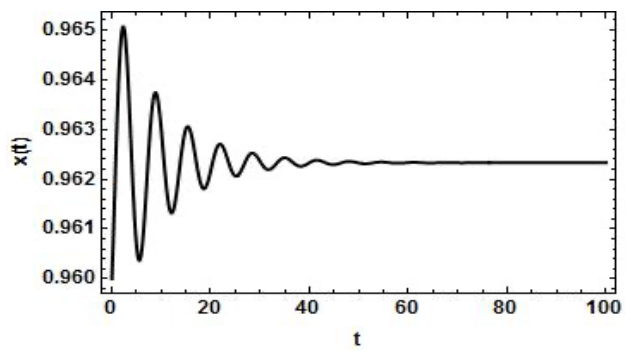

(b) Plot of $x(t)$ for system (3)

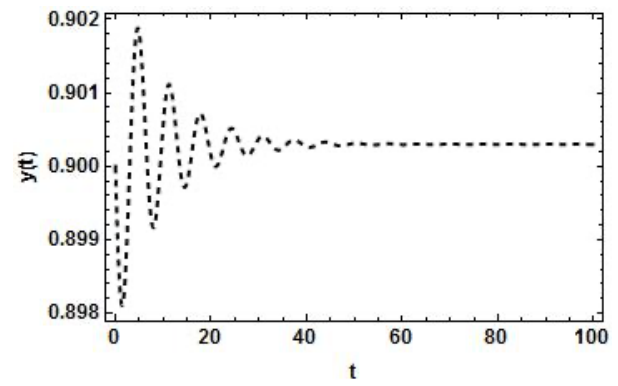

(c) Plot of $\mathrm{y}(t)$ for system (3)

Figure 11. Phase portrait and plots for system (3) with $(r, \mu)=\left(\frac{1}{8}, 0.9003\right)$ and initial conditions $\mathrm{x}(0)=0.962332, \mathrm{y}(0)=0.9003$. 
On the other hand, fixing the value of $\mu=0.9003$, then system (3) undergoes Hopf bifurcation at $r=\frac{1}{2}\left(-1-2 \mu^{2}+\sqrt{1+8 \mu^{2}}\right)=0.0573342390229552$.

At $\mu=0.9003$ and $r=0.0573342390229552$, the plots for system (3) are depicted in Fig. 12. From Fig. 12(a), it is easy to see the appearance of closed invariant curve enclosing the positive equilibrium $(1.0373621731772493,0.9003)$.

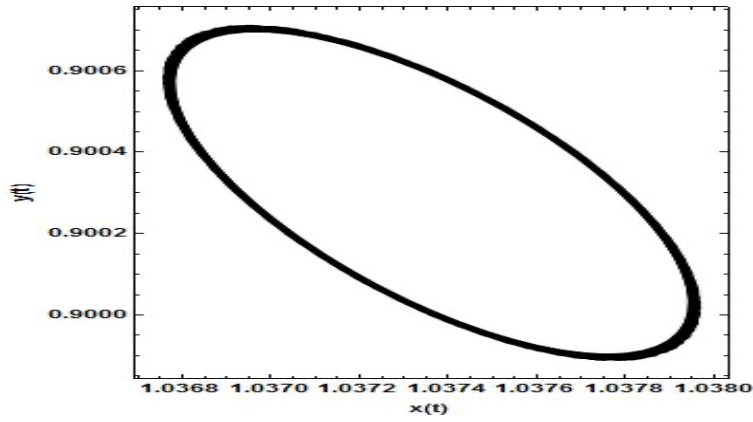

(a) Phase portrait for system (3)

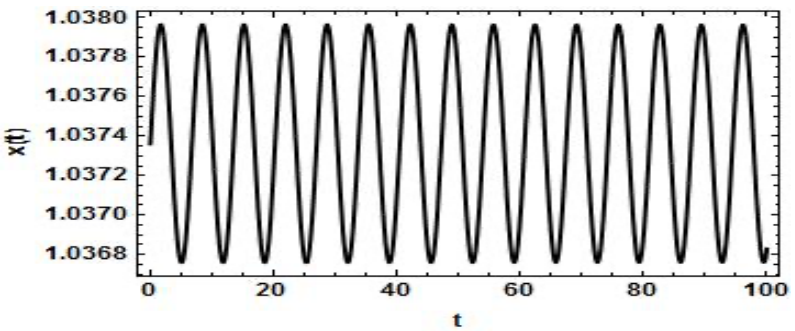

(b) Plot of $x(t)$ for system (3)

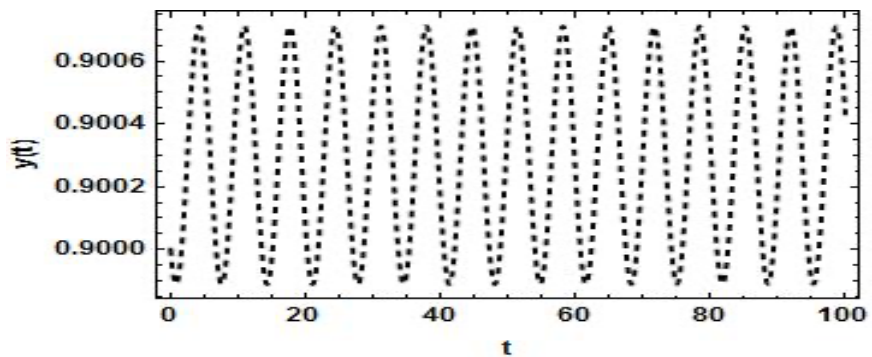

(c) Plot of $\mathrm{y}(t)$ for system (3)

Figure 12. Phase portrait and plots for system (3) with $(r, \mu)=(0.057334239,0.9003)$ and initial conditions $\mathrm{x}(0)=1.03736, \mathrm{y}(0)=0.9003$. 
In order to develop the dynamical consistency between systems (3) and (4), we choose exactly similar parametric values for both continuous and discrete models except the step size $h=$ 0.295775 , for the discrete model. In this case, the absolute difference between $r$ and $r_{1}$ is $\left|r-r_{1}\right|=0.06766582769926094$. The variation between $\left|r-r_{1}\right|$ and $h$ is given in Table 1. From Table 1 it is obvious that for smaller step size $h$ the critical values of bifurcation parameter $r$ for the emergence of a Hopf bifurcation and a Neimark-Sacker bifurcation are nearly identical, that is, $\left|r-r_{1}\right| \rightarrow 0$ as $h \rightarrow 0$.

Table 1. Variation of $r_{1}$ and $\left|r_{1}-r\right|$ with different values of $h$.

\begin{tabular}{|c|c|c|}
\hline$h$ & $r$ & $\left|r-r_{1}\right|$ \\
\hline 0.000001 & 0.05733447799855375 & $2.389755985460207 \times 10^{-7}$ \\
\hline 0.01 & 0.059720566559284406 & 0.002386327536329204 \\
\hline 0.2 & 0.10378691697971919 & 0.046452677956763985 \\
\hline 0.5 & 0.1677999862528797 & 0.1104657472299245 \\
\hline 0.8 & 0.22449769400031547 & 0.16716345497736027 \\
\hline 0.9 & 0.24179367857225356 & 0.18445943954929836 \\
\hline
\end{tabular}

\section{Conclusion}

A cubic autocatalator chemical reaction model is considered for its discretization and qualitative analysis. The discretization is performed by considering a consistency preserving scheme. For this, a nonstandard finite difference scheme is implemented to obtain a discrete counterpart of the given model. Our investigation reveals that continuous system undergoes Hopf bifurcation about its coexistence whenever $r$ is taken as a bifurcation parameter, and it passes through a critical value $r \equiv r_{0}=\frac{1}{2}\left(-1-2 \mu^{2}+\sqrt{1+8 \mu^{2}}\right)$ such that $0<\mu<1$. Moreover, the first Lyapunov exponent is computed in the closed form given as follows:

$$
L=\frac{3\left(\sqrt{1+8 \mu^{2}}-1\right)-4 \mu^{2}\left(1+\sqrt{1+8 \mu^{2}}\right)}{32 \mu^{2}} .
$$

On the other hand, the discrete counterpart, which is obtained via a nonstandard finite scheme, undergoes Neimark-Sacker bifurcation around its coexistence as step size $h$ is taken as a bifurcation parameter, and it passes through the critical value $h \equiv h_{1}=1+\frac{2 r}{\mu^{2}-r}-\frac{1}{r+\mu^{2}}$. The conditions for the existence and direction of Neimark-Sacker bifurcation are given in Theorem 
3.1. Numerical simulation reveals that our discretization is bifurcation preserving, and even with smaller step size the first Lyapunov exponents are identical in both cases.

In order to discuss comprehensive rich dynamical behavior of another discrete counterpart of cubic autocatalator chemical reaction model, one may apply Euler approximation to system (3). In case of Euler approximation, one may discuss some additional types of bifurcations and chaos control. For such types of discussions, we refer to [44-50] and reference therein. Discretization of system (3) through Euler approximation, bifurcation analysis and chaos control for the obtained model will be our future task.

\section{References}

[1] Q. Din, T. Donchev, D. Kolev, Stability, bifurcation analysis and chaos control in chlorine dioxide-iodine-malonic acid reaction, MATCH Commun. Math. Comput. Chem. 79 (2018) 577-606.

[2] J. M. L. Corbel, J. N. J. Van Lingen, J. F. Zevenbergen, O. L. J. Gijzeman, A. Meijerink, Strobes: pyrotechnic compositions that show a curious oscillatory combustion, Angew. Chem. Int. Ed. 52 (2013) 290-303.

[3] M. Chaplin, C. Bucke, Enzyme Technology, Cambridge Univ. Press, Cambridge, 1990.

[4] A. B. Cunningham, J. E. Lennox, R. J. Ross (Eds.), Biofilms: The Hypertextbook, 2010, https://www.cs.montana.edu/webworks/projects/stevesbook/, accessed 2018-10-13.

[5] R. J. Field, R. M. Noyes, Oscillations in chemical systems. IV. Limit cycle behavior in a model of a real chemical reaction. J. Chem. Phys. 60 (1974) 1877-1884.

[6] P. Gray, S. R. Kay, S. K. Scott, Oscillations of an exothermic reaction in a closed system. I. Approximate (exponential) representation of Arrhenius temperature-dependence, Proc. R. Soc. Lond. A. 416 (1988) 321-341.

[7] B. F. Gray, M. J. Roberts, P. Gray, Analysis of chemical kinetic systems over the entire parameter space I. The Sal'nikov thermokinetic oscillator, Proc. R. Soc. Lond. A. 416 (1988) 391-402.

[8] S. R. Kay, S. K. Scott, Oscillations of simple exothermic reactions in a closed system. II. Exact Arrhenius kinetics, Proc. R. Soc. Lond. A. 416 (1988) 343-359.

[9] P. Gray, S. K. Scott, Autocatalytic reactions in the isothermal, continuous stirred tank reactor: Oscillations and instabilities in the system $\mathrm{A}+2 \mathrm{~B} \rightarrow 3 \mathrm{~B} ; \mathrm{B} \rightarrow \mathrm{C}$, Chem. Eng. Sci. 39 (1984) 1087-1097.

[10] L. K. Forbes, C. A. Holmes, Limit-cycle behaviour in a model chemical reaction: the cubic autocatalator, J. Eng. Math. 24 (1990) 179-189.

[11] J. H. Merkin, D. J. Needham, S. K. Scott, Oscillatory chemical reactions in closed vessels, Proc. R. Soc. Lond. A. 406 (1986) 299-323. 
[12] J. H. Merkin, D. J. Needham, S. K. Scott, On the creation, growth and extinction of oscillatory solutions for a simple pooled chemical reaction scheme, SIAM J. Appl. Math. 47 (1987) 1040-1060.

[13] B. F. Gray, R. A. Thuraisingham, The cubic autocatalator: the influence of degenerate singularities in a closed system, J. Eng. Math. 23 (1989) 283-293.

[14] J. D. Murray, Mathematical Biology II, Spatial Models and Biomedical Applications, Springer, New York, 2001.

[15] R. P. Agarwal, P. J. Y. Wong, Advance Topics in Difference Equations, Kluwer, Dordrecht, 1997.

[16] R. Kapral, Discrete models for chemically reacting systems, J. Math. Chem. 6 (1991) 113-163.

[17] R. K. Pearson, Discrete-Time Dynamic Models, Oxford Univ. Press, Oxford, 1999.

[18] C. A. Floudas, X. Lin, Continuous-time versus discrete-time approaches for scheduling of chemical processes: a review, Comput. Chem. Eng. 28 (2004) 2109-2129.

[19] Z. U. Zafar, K. Rehan, M. Mushtaq, M. Rafiq, Numerical treatment for nonlinear Brusselator chemical model, J. Differ. Eq. Appl. 23 (2017) 521-538.

[20] A. Sanayei, Controlling chaotic forced Brusselator chemical reaction, Proc. World Congress Engin. 3 (2010).

[21] L. Xu, L. J. Zhao, Z. X. Chang, J. T. Feng, G. Zhang, Turing instability and pattern formation in a semi-discrete Brusselator model. Mod. Phys. Lett. B. 27 (2013) \#1350006.

[22] P. Yu, A. B. Gumel, Bifurcation and stability analyses for a coupled Brusselator model, J. Sound Vib. 244 (2001) 795-820.

[23] A. A. Golovin, B. J. Matkowsky, V. A. Volpert, Turing pattern formation in the Brusselator model with superdiffusion, SIAM J. Appl. Math. 69 (2008) 251-272.

[24] S. H. Strogatz, Nonlinear Dynamics and Chaos with Applications to Physics, Biology, Chemistry, and Engineering, Addison-Wesley, New York, 1994.

[25] X. S. Luo, G. Chen, B. H. Wang, J. Q. Fang, Hybrid control of period-doubling bifurcation and chaos in discrete nonlinear dynamical systems, Chaos Soliton Fract. 18 (2003) 775-783.

[26] J. Guckenheimer, P. Holmes, Nonlinear Oscillations, Dynamical Systems, and Bifurcations of Vector Fields, Springer, New York, 1983.

[27] C. Robinson, Dynamical Systems: Stability, Symbolic Dynamics and Chaos, CRC Press, Boca Raton, 1999.

[28] S. Wiggins, Introduction to Applied Nonlinear Dynamical Systems and Chaos, Springer, New York, 2003.

[29] Y. H. Wan, Computation of the stability condition for the Hopf bifurcation of diffeomorphisms on $R^{2}$, SIAM J. Appl. Math. 34 (1978) 167-175.

[30] Q. Din, Bifurcation analysis and chaos control in discrete-time glycolysis models. $J$. Math. Chem. 56 (2018) 904-931. 
[31] L. A. Leach, J. H. Merkin, S. K. Scott, Two-cell coupled cubic autocatalator: the effect of the uncatalysed reaction, Dyn. St. Sys. 7 (1992) 245-266.

[32] L. K. Forbes, Limit-cycle behaviour in a model chemical reaction: the Sal'nikov thermokinetic oscillator, Proc. R. Soc. Lond. A. 430 (1990) 641-651.

[33] H. I. Abdel-Gawad, K. M. Saad, On the behaviour of solutions of the two-cell cubic autocatalator reaction model, ANZIAM. J. 44 (2002) E1-E32.

[34] Q. Zhao, H. Xu, L. Tao, Homogeneous-heterogeneous reactions in boundary-layer flow of a nanofluid near the forward stagnation point of a cylinder, J. Heat Trans. 139 (2017) \#034502.

[35] A. A. Alderremy, K. M. Saad, J. F. Gómez-Aguilar, S. Aly, D. Kumar, J. Singh, New models of fractional blood ethanol and two-cell cubic autocatalator reaction equations, Math. Methods. Appl. Sci., in press, doi: $10.1002 / \mathrm{mma} .7188$.

[36] A. Uppal, W. H. Ray, A. B. Poore, The classification of the dynamic behavior of continuous stirred tank reactors-influence of reactor residence time, Chem. Eng. Sci. 31 (1976) 205-214.

[37] A. Mahecha-Botero, P. Garhyan, S. S. E. H. Elnashaie, Non-linear characteristics of a membrane fermentor for ethanol production and their implications, Nonlin. Anal. Real World Appl. 7 (2006) 432-457.

[38] L. N. Sridhar, Elimination of oscillations in fermentation processes, AIChE J. 57 (2011) 2397-2405.

[39] N. Zhang, W. D. Seider, B. Chen, Bifurcation control of high-dimensional nonlinear chemical processes using an extended washout-filter algorithm, Comput. Chem. Eng. 84 (2016) 458-481.

[40] C. A. PérezGómez, J. Espinosa, Design method for continuous bioreactors in series with recirculation and productivity optimization. Chem. Eng. Res. Des. 137 (2018) 544-552.

[41] X. Zhao, W. Marquardt, Reactor network synthesis with guaranteed robust stability, Comput. Chem. Eng. 86 (2016) 75-89.

[42] C. Zhai, A. Palazoglu, S. Wang, W. Sun, Strategies for the analysis of continuous bioethanol fermentation under periodical forcing, Ind. Eng. Chem. Res. 56 (2017) 3958-3968.

[43] M. E. E. Abashar, S. S. E. H. Elnashaie, Multistablity, bistability and bubbles phenomena in a periodically forced ethanol fermentor, Chem. Eng. Sci. 66 (2011) 6146-6158.

[44] Q. Din, A novel chaos control strategy for discrete-time Brusselator models, J. Math. Chem. 56 (2018) 3045-3075.

[45] Q. Din, K. Haider, Discretization, bifurcation analysis and chaos control for Schnakenberg model, J. Math. Chem. 58 (2020) 1615-1649.

[46] Q. Din, M. I. Khan, A discrete-time model for consumer-resource interaction with stability, bifurcation and chaos control, Qual. Theory Dyn. Syst. 20 (2021) 1-35. 
[47] S. S. Zhou, H. Jahanshahi, Q. Din et al., Discrete-time macroeconomic system: Bifurcation analysis and synchronization using fuzzy-based activation feedback control, Chaos Soliton Fract. 142 (2021) \#110378.

[48] Q. Din, N. Saleem, M. S. Shabbir, A class of discrete predator-prey interaction with bifurcation analysis and chaos control, Math. Model. Nat. Phenom. 15 (2020) 60.

[49] Q. Din, Stability, bifurcation analysis and chaos control for a predator-prey system, $J$. Vib. Control. 25 (2019) 612-626.

[50] Q. Din, Complexity and chaos control in a discrete-time prey-predator model, Commun. Nonlin. Sci. Num. Sim. 49 (2017) 113-134. 